\title{
The Role of Addictive Behaviors in Childhood Obesity
}

\author{
Heather L. Yardley • Jacquelyn Smith • \\ Carolyn Mingione $\cdot$ Lisa J. Merlo
}

Published online: 28 February 2014

(C) Springer International Publishing AG 2014

\begin{abstract}
Overweight and obesity have risen substantially in the last few decades. Recently, food addiction has gained attention as a possible explanation for this increase. The purpose of this paper is to review the evidence for food addiction and its role in the rise of overweight and obesity in youth. Diagnostic symptoms, prevalence, and neurobiology of food addiction are reviewed. An overview of treatment options for youth is provided. Additional research on the nature of food addiction in youth as well as continued investigation of factors related to the rise in obesity is warranted.
\end{abstract}

Keywords Food addiction · Youth · Addictive behaviors . Childhood obesity $\cdot$ Treatment options

\section{Introduction}

Childhood obesity has risen over the last few decades and continues to be the leading health-related concern for youth

\author{
H. L. Yardley $(\bowtie)$ \\ Division of Pediatric Psychology and Neuropsychology, \\ Nationwide Children's Hospital, 700 Children's Drive, Columbus, \\ OH 43205, USA \\ e-mail: Heather.Yardley@NationwideChildrens.org \\ J. Smith \\ Psychiatry and Behavioral Medicine Center, \\ Children's Hospital of Wisconsin, 9000 W. Wisconsin Ave, \\ Milwaukee, WI 53226, USA \\ e-mail: jsmith@chw.orf \\ C. Mingione \\ Department of Psychology, University of Cincinnati, 2600 Clifton \\ Ave, Cincinnati, OH 45220, USA \\ e-mail: mingiocn@mail.uc.edu

\section{J. Merlo} \\ Department of Psychiatry, University of Florida, 4001 NW 13th St, \\ Gainesville, FL 32608, USA \\ e-mail: Lmerlo@ufl.edu
}

[1-6]. Obesity has been linked to health problems; for example, being overweight in youth has been shown to place individuals at greater risk for pulmonary difficulties, cancer, and type II diabetes mellitus [7, 8]. In terms of psychosocial adjustment, youth who are overweight suffer difficulties related to quality of life [9], teasing [10], and overall emotional distress $[11,12]$. Further, youth who are overweight may become overweight adults [13] and continue to experience weight-related concerns for health and well-being.

Numerous explanations for the rise in obesity have been proposed, including availability of high fat/calorie and hyperpalatable foods [14-16], biological tendencies to save food for times of famine coupled with consumption of easily available food [17], and more recently, food addiction [18, 19•, 20•]. Food addiction has garnered attention in popular culture (websites such as Food Addicts Anonymous, symptom checker on WebMD) as well as in scientific research [21-23]. Generally, addiction involves compulsion to engage in a behavior (i.e., take a substance), loss of control in limiting behavior/substance use, and a negative emotional state if denied behavior/substance use [24]. It is important to understand the difference between occasional excessive use versus pathological use of substances necessary for survival [17].

In humans, there is evidence that disordered eating-related behaviors (i.e., binge-eating disorder) are associated with neurobiological changes similar to those found in other addictions [25-27]. In particular, the neurotransmitter dopamine (DA) has been implicated in developing addictions, including food addiction, as the brain's reward system does not differentiate between a 'reward' from a substance or a behavior [28]. Increases in DA are also associated with initiation of and participation in drug-seeking behaviors [29, 30]. In humans, studies have demonstrated that when individuals with food addiction think about preferred foods, the nucleus accumbens releases priming doses of DA [31]. This is similar to the brain response in individuals who are drug or alcohol addicted. 
In addition to the neurological similarities between food and drug addiction, there is evidence of behavioral similarities. Animal models suggest a link between binge eating and addictive-like eating [32], such that rats exposed to hyperpalatable foods engage in binge-eating behaviors (i.e., consuming large quantities in a short time, seeking food despite negative consequences) $[15,33,34]$. In humans, individuals continue to overeat despite significant psychosocial and physical health consequences, including diabetes, heart disease, and stigmatization $[26,34]$. In a sample of obese treatment-seeking adults with binge-eating disorder, $75 \%$ reported continuing to eat despite negative consequences [35••].

There are few studies examining food addiction in youth $[36 \bullet \bullet, 37 \bullet \bullet]$ despite increasing evidence that food addiction is a significant problem. The rest of this review will present evidence for food addiction in youth, including symptoms, prevalence, relation of food addiction to obesity, and an overview of treatment in youth.

\section{Symptoms of Food Addiction in Children/Adolescents}

Operationalizing symptoms of food addiction is difficult, as no formal diagnostic criteria or accepted definitions of food addiction exist at this time. Furthermore, the examination of food addiction within the pediatric population is extremely limited.

The majority of research on food addiction has occurred in the adult population and utilizes modified diagnostic criteria for substance dependence from the DSM-IV-TR [38] as the basis for conceptualizing food addiction. The most widely used measure of food addiction in adults is the Yale Food Addiction Scale (YFAS), developed in 2009 by Gearhardt et al. [39]. The YFAS is a 25-item, self-report measure asking subjects food-related questions that parallel substance dependence criteria: (i) substance taken in greater quantity and for longer than intended; (ii) persistent desire or unsuccessful repeated attempts to quit; (iii) great time and effort to obtain, use, and recover from substance; (iv) involvement in important life activities stopped or reduced; (v) continued use despite problems; (vi) tolerance; (vii) withdrawal (p.432). Responses can be scored either as a symptom count, reflecting the number of dependence symptoms present, or as a diagnostic score, with food addiction being 'diagnosed' when respondents endorse three dependence symptoms plus clinical distress or impairment. Since initial validation with a sample of normal weight undergraduate students [39], the YFAS has been validated and utilized with more diverse samples including patients with binge-eating disorder [35••, 40].

To date, only two studies have addressed food addiction symptoms in childhood and adolescence. Because no validated measure of food addiction in children existed, Merlo et al. [36••] created the Eating Behaviors Questionnaire (EBQ) to assess addictive eating behaviors in a small sample of children ages 8 to 19 years who were receiving treatment for being overweight or obese. The EBQ is a 20 -item, self-report measure with parallel parent and child forms. Items were adapted from DSM-IV-TR criteria for substance abuse/dependence and focus on compulsive eating, attempts to cut down on eating, and continued eating despite consequences. Each item is scored on a 6-point Likert-type scale, with higher scores indicating greater severity of symptoms. The most commonly reported symptoms in children were related to compulsive eating and attempts to cut down, while the least commonly endorsed items symptoms were linked to interference with social/familial interactions (e.g., missing out on activities, fighting with others about eating). Not only did this study offer preliminary support for food addiction in youth, but it also established the EBQ as measure with good initial psychometric properties (internal consistency and concurrent validity) for the assessment of food addiction in child and adolescent samples.

Pretlow [37••] demonstrated additional support for food addiction in youth. Qualitative data were gathered and analyzed from 10 years of internet bulletin board posts, chat room transcripts, and poll responses from overweight respondents ages 8 to 21 . The most commonly endorsed symptoms focused on consuming large amounts of food over a long period of time, unsuccessful efforts to cut down on consumption, and continued eating despite adverse consequences. In addition, $77 \%$ of respondents reported eating more now than when they first became overweight, and $56 \%$ endorsed urges or cravings for certain foods, which may be related to tolerance and withdrawal in the pediatric sample. Specifically, youth in that study reported having to ingest larger amounts in order to feel satiated (tolerance) and feeling extreme discomfort when unable to eat a desired or craved food (withdrawal).

Recent changes to criteria and terminology for substancerelated and addictive disorders with the publication of the DSM-5 [41] may impact future food addiction research. Specifically, substance abuse and dependence criteria have been incorporated into a more encompassing diagnosis of substance use disorder. Symptoms remain relatively unchanged, but two or more criteria must be met for a diagnosis of substance use disorder, with increased symptoms reflecting greater severity of the disorder. Thus, criteria and measures of food addiction will likely need to be adjusted accordingly.

\section{Prevalence of Food Addiction in Pediatrics}

When compared with the adult literature, prevalence data on food addiction in children is limited. Until recently there were no widely used measures to assess this construct in children and, consequently, studies have used different measures and definitions to assess food addiction in children, making 
comparisons between studies difficult. Moreover, the limited number of studies and inadequate sample sizes prevent largescale prevalence estimates. However, despite these limitations, the studies discussed below provide important preliminary information regarding the clinical characteristics and prevalence of food addiction symptoms in both clinical and non-clinical samples.

In a recent study, approximately $7 \%$ of children from a community sample met diagnostic criteria for food addiction as determined by the Yale Food Addiction Scale for Children (YFAS-C), which requires three or more food addiction symptoms in addition to clinically significant impairment or distress over the last 12 months [42]. Thirty-nine percent of children in the study endorsed more than three symptoms and $10 \%$ reported clinically significant impairment or distress due to symptoms. The most commonly reported symptom was giving up activities due to overeating (i.e., "I eat so much that I feel bad afterwards. I feel so bad that I do not do things I like"). Another study examining food addiction symptoms using a scale developed for use in the study (i.e., the Children's Eating Behaviors Questionnaire; EBQ) found that approximately $15 \%$ of treatment-seeking children ages 8 19 years reported that they 'often', 'usually' or 'always' think that they are addicted to food, and an additional $17.4 \%$ 'sometimes' feel that way [36••]. The most commonly endorsed items on the EBQ were related to compulsive eating and lack of control. Finally, in a large qualitative internet study, children and teenagers between the ages of 8 and 21 years, with body mass index (BMI) greater than or equal to the 85th percentile, who were unsuccessful in losing weight, discussed addictive behaviors toward food in online chat rooms. Pretlow [37•0] noted that the behaviors described closely paralleled DSM-IV criteria for substance dependence, and he identified consuming large amounts over a long period of time, unsuccessful efforts to cut down, and continued use despite adverse consequences as the most commonly described criteria. Also of note, when teens and preteens were provided with a definition of addiction, $29 \%$ endorsed "I think I'm addicted to most foods" and $37 \%$ indicated "I'm addicted, but only to certain foods" [36••].

Thus, current research provides preliminary evidence that food addiction may be a relevant and prevalent problem in children; however, extant research is sparse. As such, there is a need for additional research in this area, specifically utilizing validated measures, larger sample sizes, and additional clinical samples (e.g., children with eating disorders or primarily obese/overweight samples).

\section{Relationship between Food Addiction and Obesity}

The prevalence of pediatric obesity has rapidly increased over the past few decades, with estimates categorizing $16.9 \%$ of youth aged 2-19 years as obese [43]. This is particularly concerning due to the numerous medical, psychological, and social comorbidities of pediatric obesity, many of which extend into adulthood [44]. Due to this growing public health concern, it is imperative to identify causes and correlates of obesity in pediatrics. Indeed, growing literature suggests that symptoms of food addiction may contribute to overweight/

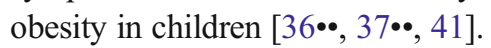

In a community sample of children, food addiction symptoms as measured by the YFAS-C were significantly positively related to higher BMI [41]. Merlo and colleagues [36••] also found a positive correlation between BMI and food addiction symptoms, which approached statistical significance $(p=0.06)$. These authors noted, however, that they had limited power $(n=40)$ and that the BMI data was heavily skewed toward the obese end of the spectrum, which may have attenuated the findings. Also of interest, Pretlow [37••] found that individuals with higher BMIs had more of a struggle with losing weight, which led him to speculate that food addiction may be on a continuum, with level of addiction increasing with BMI.

These studies also found that food addiction symptoms in children are related to other constructs associated with overweight and obesity, such as emotional eating, decreased satiety responsiveness, uncontrolled eating, food preoccupation, overconcern with body size, and decreased caloric awareness

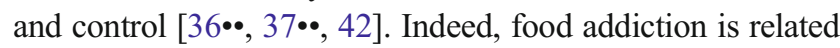
not only to BMI, but also to additional factors associated with increased risk for children being overweight or obese, highlighting the potential clinical relevance of food addiction in contributing to the obesity epidemic in children.

\section{Intervention}

Treatment for any addiction is complex and multi-faceted. To be successful, treatment must cause long-term changes in lifestyle. In substance-use treatment, the goal is abstinence from the substance; however, this is not possible in addictiveeating behavior. Thus, treatment must be focused on symptom management and relapse prevention. It is important to note that motivation for change is key to successful intervention. Individuals have to be ready and enthusiastic to make changes [45].

Cognitive behavioral therapy (CBT) is the most well established treatment for binge-eating disorder and substance abuse disorders [46], and has been used successfully in youth who are overweight (for review, see [47]). In CBT, therapists assist patients with self-monitoring (of use in this model), identifying and changing automatic thoughts related to using (cognitive restructuring), stimulus control (identifying triggers for use), developing coping strategies, goal setting, behavioral activation, and relapse prevention. One key difference in using 
CBT for eating behaviors as opposed to substance use is a lack of emphasis on abstinence. Thus, treatment must focus on helping the individual use normative guidelines for dietary requirements and exercise. There are no studies of CBT for treating food addiction directly; however, it is likely that this would improve outcomes in these individuals and warrants further investigation.

Motivational interviewing (MI) is a client-focused method to enhance motivation to change by resolving ambivalence and eliciting and strengthening personal motivation for change [48]. Motivational interviewing was initially developed for treatment of substance abuse. It has since been used to treat a variety of mental health concerns in which motivational factors are key, such as weight loss [49]. It can be used as an independent intervention or as a precursor to other interventions like CBT [50]. MI has been suggested as an intervention for youth who are overweight or obese [51]. In a study of youth aged between 10 and 18 years, Walpole and colleagues [52] reported that the addition of MI to traditional therapy for obesity increased the number of sessions attended but did not improve overall outcomes. They suggest using multiple methods of treatment in youth who are overweight.

There are numerous successful 12-step programs for individuals with substance use disorders (i.e., Alcoholics Anonymous, Narcotics Anonymous). Overeaters and Food Addicts Anonymous uses the 12-step model to address symptoms of food addictive behaviors. Participants in the program view their food addiction as similar to the experience of other substance-dependent individuals. However, the focus in this program is balanced eating rather than abstinence. Overeaters Anonymous uses the principles of anonymity in a group along with fellowship and spirituality to aid in recovery. Generally, 12-step programs are flexible in that they allow individuals to repeat steps and welcome them back into the group if a relapse occurs; thus, this model is a good fit for food-addicted individuals as relapse may occur. There is some evidence that adolescents benefit in the same ways as adults from 12-step programs [53], but no research has been conducted on 12-step programs for food addiction in youth. Additional research is needed to determine the effectiveness of this model in children and adolescents.

It is important to consider the role of diet and exercise in any of the above treatment recommendations to help ameliorate the negative effects of overweight and obesity. Generally, treatment for obesity in youth has been shown to be effective in reducing weight [54, 55]. A Cochrane review [54] suggested that while treatment is effective overall, there is significant heterogeneity amongst treatments. They suggest that the most effective treatment would be multifaceted and include incorporating education on healthy eating, activity, and self- image into the school day as well as providing support for adults (parents, teachers) who are implementing and encouraging healthy changes. A separate meta-analysis found that obesity prevention programs that target youth are brief, focus solely on weight-control behaviors, and allow participants to select the interventions that are the most effective [56]. Additionally, the role of parents within treatment should be considered. Youth making a change to diet or exercise will be more successful when parents are actively engaged in the treatment process and, as noted, have support when implementing interventions at home. Taken with the information on treatment above, clinicians will be most effective in treating food addiction and obesity when using multicomponent models and when the motivation of the participants is high.

\section{Conclusion}

Obesity and overweight continue to rise, particularly in children. One possible explanation is an increase in youth who experience symptoms of food addiction [36••, 37••]. There is growing neurological and behavioral evidence that individuals experience addiction to food in the same ways as addiction to substances. However, extant research in children and youth is sparse. Additional research examining neurobiological changes in youth would strengthen the food addiction model. Studies should incorporate larger, more diverse samples including clinical (i.e., eating disordered, primarily obese) populations to document the nature of differences.

Behaviorally, studies have demonstrated that symptoms of food addiction are correlated with other constructs that may contribute to obesity. Emotional or uncontrolled eating, preoccupation with food, and concern with body size have all been associated with symptoms of food addiction. This underscores the importance of understanding symptoms of food addiction and how they present in youth. Further, treatment programs should include components that address these symptoms directly. As noted, the most successful programs are multifaceted and additional understanding of food addiction symptoms will serve to improve our treatment outcomes.

\section{Compliance with Ethics Guidelines}

Conflict of Interest Heather L. Yardley, Jacquelyn Smith, Carolyn Mingione and Lisa J. Merlo declare they have no conflict of interest.

Human and Animal Right and Informed Consent This article does not contain any studies with human or animal subjects performed by any of the authors. 


\section{References}

Papers of particular interest, published recently have been highlighted as:

- Of importance

- Of particular importance

1. National Center for Health Statistics. Prevalence of Obesity Among Children and Adolescents: United States, Trends 1963-1965 Through 2009-2010. 2012. Retrieved January 1, 2014 from the Centers for Disease Control Web site: http://www.cdc.gov/nchs/ data/hestat/obesity_child_09_10/obesity_child_09_10.htm.

2. Ogden CL, Carroll MD, Kit BK, Flegal KM. Prevalence of obesity and trends in body mass index among US children and adolescents. J Am Med Assoc. 2012;307:483-90.

3. Parikh N, Pencina MJ, Wang TJ, Lanier KJ, Fox CS, D'Agostino $\mathrm{RB}$, et al. Increasing trends in incidence of overweight and obesity over 5 decades. Am J Med. 2007;120:242-50.

4. Shisslak CM, Crago M, McKnight KM, Estes LS, Gray N, Parnaby OG. Potential risk factors associated with weight control behaviors in elementary and middle school girls. J Psychosom Res. 1998;44: 301-13.

5. Styne DM. Childhood and adolescent obesity: prevalence and significance. Pediatr Clin N Am. 2001;48:823-54. vii.

6. Ogden CL, Flegal KM, Carroll MD, Johnson CL. Prevalence and trends in overweight among US children and adolescents, 19992000. J Am Med Assoc. 2002;288:1728-32.

7. Hill K, Pomeroy C. Assessment of physical status of children and adolescents with eating disorders and obesity. In: Thompson JK, Smolak L, editors. Body image, eating disorders, and obesity in youth: Assessment, prevention, and treatment. Washington, DC: American Psychological Association; 2001. p. 171-92.

8. Must A, Strauss RS. Risks and consequences of childhood and adolescent obesity. Int J Obes. 1999;23:s2-s11.

9. Williams J, Wake M, Hesketh K, Maher E, Water E. Health-related quality of life of overweight and obese children. J Am Med Assoc. 2005;293:70-6.

10. Neumark-Sztainer D, Falkner N, Story M, Perry C, Hannan PJ, Mulert S. Weight-teasing among adolescents: correlations with weight status and disordered eating behaviors. Int $\mathrm{J}$ Obes. 2002;26:123-31.

11. Israel AC, Ivanova MY. Global and dimensional self-esteem in preadolescent and early adolescent children who are overweight: Age and gender differences. Int $\mathrm{J}$ Eat Disord. 2002;31:424-9.

12. Mellin AE, Neumark-Sztainer D, Story M, Ireland M, Resnick MD. Unhealthy behaviors and psychosocial difficulties among overweight adolescents: The potential impact of familial factors. J Adolesc Health. 2002;31:145-53.

13. Whitaker RC, Wright JA, Pepe MS, Seidel KD, Dietz WH. Predicting obesity in young adulthood from childhood and parental obesity. N Engl J Med. 1997;337:869-73.

14. Avena NM, Gold JA, Kroll C, Gold MS. Further developments in the neurobiology of food and addiction: Update on the state of the science. Nutrition. 2012;28:341-3.

15. Avena NM, Rada P, Hoebel BG. Evidence for sugar addiction: Behavioral and neurochemical effects of intermittent, excessive sugar intake. Neurosci Biobehav Rev. 2008;32:20-39.

16. Swinburn BA, Sacks G, Hall MD, McPherson K, Finegood DT, Moodie ML, et al. The global obesity pandemic: shaped by global drivers and local environments. Lancet. 2011;378: 804-14.

17. Gold MS, Graham NA, Cocores JA, Nixon SA. Food addiction? J Addict Med. 2009;3:42-4.
18. Gearhardt AN, Davis C, Kuschner R, Brownell KD. The addiction potential of hyperpalatable foods. Curr Drug Abuse Rev. 2011;4: $140-5$.

19. Meule A. How prevalent is "food addiction"? Front Psychiatry. 2011;2:1-4. Brief review of prevalence and assessment of food addiction in youth and adults.

20. Smith DG, Robbins TW. The neurological underpinnings of obesity and binge eating: a rationale for adopting the food addiction model. Biol Psychiatry. 2013;73:804-10. Provides a review of neurological and behavioral traits related to food addiction.

21. Avena NM, Gold MS. Food and addiction - sugars, fats, and hedonic overeating. Addiction. 2011;107:1214-5.

22. Gearhardt AN, Grilo CM, DiLeone RJ, Brownell KD, Potenza MN. Can food be addictive? Public health and policy implications. Addiction. 2011;106:1208-12.

23. Rogers PJ. Obesity - is food addiction to blame? Addiction. 2011;107:1213-4.

24. Koob GF, Sanna PP, Bloom FE. Neuroscience of addiction. Neuron. 1998;21:467-76.

25. Davis C, Carter JC. Compulsive overeating as an addiction disorder: A review of theory and evidence. Appetite. 2009;53:1-8.

26. Gearhardt AN, Corbin WR, Brownell KD. Food addiction: an examination of the diagnostic criteria for dependence. J Addict Med. 2009;3:1-7.

27. Volkow ND, Wang GJ, Fowler JS, Telang F. Overlapping neuronal circuits in addition and obesity: evidence of systems pathology. Philos Transl Royal Soc B: Biol Sci. 2008;363:3191-200.

28. Holden C. 'Behavioral' addictions: Do they exist? Science. 2001;294:980-2.

29. Bardo MT, Donohew RL, Harrington NG. Psychobiology of novelty seeking and drug seeking behavior. Behav Brain Res. 1996;77: 23-43.

30. Phillips PE, Stuber GD, Heien ML, Wightman RM, Carelli RM. Subsecond dopamine release promotes cocaine seeking. Nature. 2003;422:614-8.

31. Hodgekins CC, Cahill KS, Seaphine AE, Frost-Pineda K, Gold MS. Adolescent drug addiction treatment and weight gain. J Addict Disord. 2004;23:23-37.

32. Gold MS, Frost-Pineda K, Jacobs WS. Overeating, binge eating, and eating disorders as addictions. Psychiatr Ann. 2003;33:117-22.

33. Johnson PM, Kenny PJ. Dompamine D2 receptors in addiction-like reward dysfuction and compulsive eating in obese rats. Nat Neurosci. 2010;13:635-41.

34. Volkow ND, O'Brien CP. Issues for DSM-V: Should obesity be included as a brain disorder? Am J Psychophysology. 2007;25: $38-9$.

35.• Gearhardt AN, White MA, Masheb RM, Morgan PT, Crosby RD, Grilo CM. An examination of the food addiction construct in obese patients with binge eating disorder. Int J Eat Disord. 2012;45:65763. Study demonstrating the convergent and incremental validity and internal consistency of an established adult measure of food addiction, the Yale Food Addiction Scale, in youth.

36.• Merlo LJ, Klingman C, Malasanos TH, Silverstein JH. Exploration of food addiction in pediatric patients. J Addict Med. 2009;3:26-32. Investigated presence of food addiction symptoms in 50 youth between ages 8 and 19. Demonstrated food addiction symptoms were related to overeating, emotional/uncontrolled eating, preoccupation with food, and distorted body image.

37.• Pretlow RA. Addiction to highly pleasurable food as a cause of the childhood obesity epidemic: A qualitative internet study. Eat Disord. 2011;19:295-307. Examined youth (ages 8-21) responses on internet bulletin boards and chat rooms associated with an online intervention for youth who are overweight. A significant proportion of youth endorsed symptoms of substance dependence when describing their relationship to highly pleasurable foods. 
38. American Psychiatric Association. Diagnostic and Statistical Manual of Mental Disorders-DSM-IV-TR. 4th ed. Washington, DC: American Psychiatric Association; 2000.

39. Gearhardt AN, Corbin WR, Brownell KD. Preliminary validation of the Yale Food Addiction Scale. Appetite. 2009;52:430-6.

40. Gearhardt AN, White MA, Masheb RM, Grilo CM. An examination of food addiction in a racially diverse sample of obese patients with binge eating disorder in primary care settings. Compr Psychiatry. 2013;54:500-5.

41. American Psychiatric Association. Diagnostic and Statistical Manual of Mental Disorders-DSM-V. 5th ed. Washington, DC: American Psychiatric Association; 2013.

42. Gearhardt AN, Roberto CA, Seamans MJ, Corbin WR, Brownell KD. Preliminary validation of the Yale Food Addiction Scale for children. Eat Behav. 2013;14:508-12.

43. Ogden, C., \& Carroll, M. (2010). Prevalence of obesity among children and adolescents: United States, trends 1963-1965 through 2007-2008. Retrieved from Centers for Disease Control and Prevention website: http://www.cdc.gov/obesity/data/childhood.html

44. Acosta MC, Manubay J, Levin FR. Pediatric obesity: parallels with addiction and treatment recommendations. Harvard Rev Psychiatry. 2008;16:80-96.

45. Prochaska J, DiClemente C. Stages of change in the modification of problem behaviors. Prog Behav Modif. 1992;28:183-218.

46. Wilson GT, Grilo CM, Vitousek KM. Psychological treatments of eating disorders. Am Psychol. 2007;72:199-216.

47. Michael JC, Belendiuk KA, Kuchera AM, Rofey DL. Cognitive behavioral therapy for weight management. Behav Ther. 2013;36: 28-38.
48. Miller WR, Rollnick S. Motivational interviewing: Preparing people for change. 2nd ed. New York: Guilford Press; 2002.

49. Dunn EC, Neighbors C, Larimer ME. Motivational enhancement therapy and self-help treatment for binge eaters. Psychol Addict Behav. 2006;20:44-52.

50. Wilson GT. Psychological treatment of eating disorders. Annu Rev Clin Psychol. 2005;1:439-65.

51. Mehlenbeck RS, Wember YM. Motivational interviewing and pediatric obesity. In: Jelalian E, Steele RG, editors. Handbook of Childhood and Adolescent Obesity. New York: Springer Science and Business Media; 2008. p. 405-24.

52. Walpole B, Dettmer E, Morrongiello BA, McCrindle BW, Hamilton J. Motivational interviewing to enhance self-efficacy and promote weight loss in overweight and obese adolescents. J Pediatr Psychol. 2013;38:944-53.

53. Kelly JF, Urbanoski K. Youth recovery contexts: the incremental effects of 12-step attendance and involvement on adolescent outpatient outcomes. Alcohol Clin Exp Res. 2012;36:1219-29.

54. Waters E, de Silva-Sanigorski A, Burford BJ, Brown T, Campbell $\mathrm{KJ}$, Gao Y, et al. Interventions for preventing obesity in children (review). Cochrane Collab. 2011;12:1-212.

55. Wilfley DE, Kolko RP, Kass AE. Cognitive-behavioral therapy for weight management and eating disorders in children and adolescents. Child Adolesc Psychiatr Clin N Am. 2011;20: 271-85.

56. Stice E, Shaw H, Marti CN. A meta-analytic review of obesity prevention programs for children and adolescents: the skinny on interventions that work. Psychol Bull. 2006;132: 667-91. 